\section{In the mood for change: shifting the paradigm of mental health care in athletes-an AMSSM thematic issue}

\author{
Ashwin L Rao, ${ }^{1}$ Eugene S Hong ${ }^{2}$
}

\begin{abstract}
Mental health is an increasingly recognised and discussed topic in the sports community. To compete successfully is a challenge as athletes must prevent and manage injuries, perform academically and contend with both internal and external emotional pressures. ${ }^{12}$ These stressors can lead to disruptive thoughts and maladaptive behaviour patterns. ${ }^{3-6}$ There are an increasing proportion of young people facing mental illness. ${ }^{78}$

Media reports of tragic deaths involving athletes, who are perceived to be among the healthiest members of their community, highlight the struggles that athletes face in the shadows of perceived emotional and physical strength. There is an increasing recognition of depression and suicide, disordered eating, substance abuse, hazing and bullying, the psychological consequences of injury as real concerns in the athletic community. Yet, most providers lack of familiarity with these topics and may not be comfortable in managing the athlete expressing these concerns. ${ }^{9}$
\end{abstract}

\section{EXPERT OPINION AND FUTURE DIRECTIONS}

This AMSSM theme issue assembles a group of experts in the field of sports mental health to identify current challenges, barriers, and progress through a series of informative reviews and editorials. Dr Alex Diamond describes hazing, bullying and related consequences in athletes (see page 149). Dr Margot Putukian reviews the consequences of injury to an athlete's mental health (see page 145). Elizabeth Joy and colleagues evaluate current concepts regarding disordered eating in athletes (see page 154). These comprehensive reviews are supplemented by a series of editorial contributions. Dr Jim Bauman contemplates the historic stigma of mental health in athletic culture and measures being taken to change

\footnotetext{
${ }^{1}$ Department of Family Medicine, University of Washington, Seattle, Washington, USA; ${ }^{2}$ Department of Family, Community and Preventive Medicine, University College of Medicine, Philadelphia, Pennsylvania, USA

Correspondence to Dr Ashwin L Rao, Department of Family Medicine, University of Washington, UW Husky Stadium Sports Medicine Center 3800 Montlake Blvd NE Box 354060, Seattle, WA 98195,USA; ashwin@uw.edu
}

the culture around mental health (see page 135). Dr EH and Dr AR, the issue's editors, explore the current concerns of depression and suicidality in the college student athletes, identifying mental health as an underserved area of care (see page 136). Dr Anthony Kontos and colleagues review the burgeoning topic of concussion and its mental health consequences (see page 139). Dr Tom Trojian discusses screening tools that may be employed in the office practice to better prepare providers to identify athletes in need of mental health services (see page 137).

In one of three original research articles, Dr Irfan Asif and colleagues investigate the psychological response to being diagnosed with a potentially lethal cardiac disease (see page 163). Dr Ashwin Rao and colleagues evaluate the incidence of homicide-related death in National Collegiate Athletic Association athletes (see page 172), while Dr EH and colleagues evaluate the prevalence of clinically elevated depression symptoms in collegiate athletes (see page 167). These articles represent exciting directions that the sports medicine community is taking to better understand the scope of mental concerns in the athletes whom we treat.

This edition also highlights two articles from the $B M J$, a practice guideline regarding the management of depression in the young (see page 184) and a research manuscript that evaluates peer victimisation during adolescents and its downstream consequences (see page 176). Both articles have great value to the readership and provider interested in mental health concerns in the young.

We hope that this theme issue will serve as a springboard to better understand mental health challenges in the athletic population. Though scholarship, discussion and collaboration, sports medicine providers can foster a culture in which mental and physical concerns of athletes can be treated with equal attention.

\section{AMSSM ANNUAL MEETING: $16-20$ APRIL, 2016 DALLAS}

Our attention shifts to Dallas, Texas for the 2016 Annual meeting-April 16 through April 20th. The AMSSM Annual meeting is the largest assembly of primary care sports medicine providers, and this year's meeting highlights 25 years of AMSSM's service to the sports medicine community with a 'Back to the Future' theme. Many of AMSSM's founding members are set to introduce sessions and provide perspectives gained through time and service. The programme features notable domestic and international speakers, including NASA astronaut Dr Andrew Morgan, renowned tendon scholar Dr Hakan Alfredson, international travelling fellow Dr Maurizio Casasco, Society for Tennis Medicine and Science exchange speaker Dr Kathleen Stroia, ultrasound authority Dr Jon Jacobson, ACSM exchange lecturer Dr Blaise Williams and AOSSM exchange speaker Dr David Geier.

The meeting will also feature the introduction of the AMSSM Position Statement on Cardiovascular Screening in Athletes, hosted by past-presidents Dr Jonathan Drezner and Dr Francis O'Connor. Dr Margot Putukian will provide a review of the team physician consensus statement on illness and injury prevention. Both topics are arenas of great interest to a majority of the membership and these presentations bring cohesion to much discussed topics.

The conference is supplemented by AMSSM basic and advanced musculoskeletal ultrasound courses, a faculty development workshop, the annual SMART course and multiple supplemental morning sessions. Make sure to attend the research and case presentations to see the latest scholarship efforts of your colleagues. Please support the AMSSM Foundation project, which will be hosted on the day prior to the conference and affords another opportunity for service as an AMSSM member.

We hope that you enjoy this AMSSM-edited theme issue of BJSM. You can follow the AMSSM on Twitter (@TheAMSSM), and be keep up to date on the latest news at http://www.amssm.org.

Twitter Follow Ashwin Rao at @badash13

Competing interests None declared.

Provenance and peer review Not commissioned; externally peer reviewed.

To cite Rao AL, Hong ES. Br I Sports Med 2016;50:133-134.

Accepted 28 December 2015

Br J Sports Med 2016;50:133-134 doi:10.1136/bjsports-2015-095924 


\section{REFERENCES}

1 Wolanin A, Gross M, Hong E. Depression in athletes: prevalence and risk factors. Curr Sports Med Rep 2015;14:56-60.

2 Rao AL, Asif IM, Drezner JA, et al. Suicide in National Collegiate Athletic Association (NCAA) Athletes: a 9-year analysis of the NCAA Resolutions Database. Sports Health 2015;7:452-7.

3 Bovard RS. Risk behaviors in high school and college sport. Curr Sports Med Rep 2008;7: 359-66.
4 Dvjorak RD, Lamis DA, Malone PS. Alcohol use, depressive symptoms, and impulsivity as risk factors for suicide proneness among college students. J Affect Disord 2013;149:326-34.

5 Kokotailo PK, Henry BC, Koscik RE, et al. Substance use and other health risk behaviors in collegiate athletes. Clin J Sports Med 1996;6: 183-9.

6 Nattiv A, Puffer J. Lifestyle and health risks of collegiate athletes. J Fam Pract 1991;33: 585-90.
7 US Department of Health and Human Services. FastStats-Depression. http://www.cdc.gov/nchs/ fastats/depression.htm (accessed Sep 2015).

8 Neal TL, Diamond AB, Goldman S, et al. Interassociation recommendations for developing a plan to recognize and refer student-athletes with psychologic concerns at the collegiate level: an executive summary of a consensus statement. J Ath/ Train 2013:48:716-20.

9 Mann BJ, Grana WA, Indelicato PA, et al. A survey of sports medicine physicians regarding psychological issues in patient-athletes. Am J Sports Med 2007;35:2140-7. 